QMW-PH-98-20

Revised Version

\title{
Orbifold Duality Symmetries and Quantum Hall systems
}

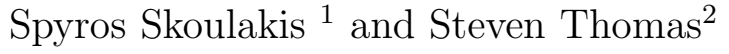 \\ Department of Physics, Queen Mary and Westfield College, \\ Mile End Road, London E1 U.K.
}

\begin{abstract}
We consider the possible role that chiral orbifold conformal field theories may play in describing the edge state theories of quantum Hall systems. This is a generalization of work that already exists in the literature, where it has been shown that $1+1$ chiral bosons living on a $n$-dimensional torus, and which couple to a $U_{1}$ gauge field, give rise to anomalous electric currents, the anomaly being related to the Hall conductivity. The well known $O(n, n ; Z)$ duality group associated with such toroidal conformal field theories transforms the edge states and Hall conductivities in a way which makes interesting connections between different theories, e.g. between systems exhibiting the integer and fractional quantum Hall effect. In this paper we try to explore the extension of these constructions to the case where such bosons live on a $n$-dimensional orbifold. We give a general formalism for discussing the relevant quantities like the Hall conductance and their transformation under the duality groups present in orbifold compactifications. We illustrate these ideas by presenting a detailed analysis of a toy model based on the two-dimensional $Z_{3}$ orbifold. In this model we obtain new classes of filling fractions, which generally correspond to fermionic edge states carrying fractional electric charge. We also consider the relation between orbifold edge theories and Luttinger liquids (LL's), which in the past have provided important insights into the physics of quantum Hall systems.
\end{abstract}

\footnotetext{
${ }^{1}$ email: s.skoulakis@qmw.ac.uk

${ }^{2}$ email: s.thomas@qmw.ac.uk
} 


\section{Introduction}

In recent years there has been a growing body of literature which has revealed that duality symmetries, in their various forms, seem to play an important role in understanding connections between the various quantum Hall hierarchies [1]-[4]. These ideas appear to fall into two approaches. In the first [5] (see also [6] for a more recent discussion and references therein), one introduces a complex Hall conductivity $\tau=\sigma_{x y}+i \sigma_{x x}$ (where $\sigma_{x x}, \sigma_{x y}$ are the longitudinal and transverse components of the conductivity tensor) which lives in the upper half plane and naturally transforms under the modular group $S L(2 ; Z)$. This complex conductivity enters as a parameter of an effective field theory that encodes localization in the quantum Hall system, and which is invariant under the modular group. On Hall plateau's, $\sigma_{x x}=0$ and $\sigma_{x y}=\nu=p / q$ in units of $\frac{e^{2}}{h}$. Under the assumption that the Renormalization Group trajectories of $\tau$ "commute" with certain subgroups of $S L(2 ; Z)$, a number of properties of both the integer quantum Hall effect (IQHE) and fractional quantum Hall effect (FQHE) (at zero temperature) can be derived [5], [6]. This includes derivation of critical values of $\tau$ as well as various universal scaling exponents. These results follow from the assumption that the quantum Hall phase diagram comes as a consequence of the action of the congruence subgroup $\Gamma_{0}(2)$ on the complex conductivity $\tau$.

Another approach to the problem is based on the well established connection between quantum Hall systems and Chern-Simons theories on the interior of the disc [7], [8] . This equivalence holds for the particularly interesting Laughlin states with filling

fractions $\nu=\frac{1}{m}, m \in 2 Z+1$ and its generalizations to rational $\nu$ as well as to the hierarchy of Jain [3]. Because of the topological nature of Chern-Simons theories they have observables only lying on the edge of the disc, which can be shown to be equivalent to a system of chiral bosons living on this boundary [7]-[9]. When such bosonic theories are coupled to a background electromagnetic field, the corresponding currents have been shown to be anomalous, with anomaly proportional to the Hall conductivity [4], [11]-[13] and hence leads to a natural correspondence with the boundary Hall current of the bulk system. Such an identification requires the bosonic edge theory to be chiral, and so for $n$ such bosons, it is natural to regard them as analogous to the coordinates of a string compactified on a $n$-dimensional torus $T^{n}$, but with additional constraint that the underlying conformal field theory be rational. It is now well established that toroidally compactified string theories possess so called $T$-duality 
symmetries which act on the various compactified background fields through which the string propagates, $\left(G_{i j}\right.$ and $B_{i j}$ in the simplest cases), and which leave the spectrum of states invariant. In the case of strings on $T^{n}$ the corresponding duality group is $O(n, n ; Z)[14]$.

The authors in [15] have showed how such string dualities can be used to generate various quantum Hall hierarchies e.g. those due to Haldane and Jain, by choosing particular values for the background fields which give Laughlin's fractions, and then transforming with certain elements of $O(n, n ; Z)$ to obtain new filling fractions $\nu^{\prime}$. Since the spectrum is invariant under these maps, one concludes that fractions related this way have the same spectrum which is particularly interesting. Furthermore this approach allows a simple construction of the wavefunctions in Haldane's hierarchy.

In this paper, pushing the stringy picture one step further, we consider the possible role of orbifold duality symmetries in the quantum Hall effect. Orbifolds are important kinds of compactifications in string theory, and are obtained by taking the quotient $T^{n} / P$ where $P$ is some discrete isometry group of the torus. Effectively, taking the quotient modifies the topology of the torus and leads to new kinds of string states [16]. At the same time, the duality symmetries present after this process are subgroups, in general, [18], of $O(n, n ; Z)$ of the torus. (Here we will assume we are dealing with symmetric orbifolds based on underlying left-right symmetric toroidal compactifications.) This partial breaking of toroidal duality symmetries can be understood from the fact that there are geometrical constraints on the background fields $G_{i j}, B_{i j}$ etc. that ensure they allow the existence of the discrete isometry group $P$. We shall see later, it is these new constraints that directly control the kinds of new filling fractions one can get following the procedures presented in [15] which we discussed above.

Before moving on, it is worth noting that it has recently been shown [27] that one can may also find interesting new fractions by using so called mirror symmetries of the toroidally compactified chiral boson theory ( or its Chern-Simons formulation). Such mirror symmetries are different in general, from the " $\mathrm{T}$ "-duality symmetries we have been discussing and their existence in various string compactifications has been well established (see e.g. the third reference in [14] ). Indeed mirror symmetry has been an important tool in understanding string compactifications on so called Calabi-Yau manifolds. On the other hand, orbifolds are well known to encode much of the topology of such manifolds including properties such as mirror symmetry . One might expect then, that the idea of using mirror symmetry to generate new filling fractions or make 
connections between those that are known, could also be applied to the orbifold edge models we consider.

The paper is organized as follows. In section 2 we briefly review the general formalism of orbifold edge theories obtained by quotienting $T^{n}$ with discrete isometries, concentrating on those aspects that will be relevant to the QHE. We show how the resulting theory may be coupled to a $1+1$ dimensional $U_{1}$ gauge field. We shall see how the basic requirement of chirality in these models can be made consistent with the quotienting procedure, and consider the general criterion, considered in [15], for the existence of fermionic edge states carrying charge $-e$. Implicit equations that define the orbifold duality symmetries are also presented. Also we discuss briefly how one can realize the orbifold construction within the Chern-Simons framework for describing chiral bosons. In section 3 we consider possible fermionic interpretations of orbifold edge theories. In the case of a single chiral boson, it is known that the corresponding fermionic picture involves the so called chiral Luttinger liquids (CLL), [2], [21] (based on the Luttinger model [34]), which in turn are related to the $1+1$ dimensional Thirring model (see e.g.[17]). Generalizations of this to the case of $n$ chiral bosons lead one to consider $n$-flavour Thirring models with $n$ complex fermions coupled to a $U_{1}$ gauge field. The orbifold edge theories we consider, should be equivalent to quotienting out this $n$-flavour Thirring model by some discrete symmetry.

In section 4 we present a simple toy model based on the two-dimensional $Z_{3}$ orbifold. Although this does not have all the physical aspects of quantum Hall systems we would like, it does illustrate some of the features that one may expect in more general examples, whilst having the advantage of being relatively simple to calculate with. We show how one can accommodate Haldane's hierarchy $\nu=\frac{1}{m} \quad m \in 2 Z+1$ within this model and then use this as a starting point to obtain other fractions using the surviving duality symmetries.

Finally in section 5 we end with some comments and conclusions.

\section{Duality symmetries in chiral orbifold edge theo- ries}

In this section we want to review the relevant orbifold constructions that have appeared in the string literature [16]. Although this is a large and well developed subject, we will 
focus our attention on the specific issues of how duality symmetries manifest themselves on the zero modes of the chiral boson edge theory, as this is the central tool we will use to investigate the possible new filling fractions we can obtain in this system. In this respect, we will follow, more or less, the notation in [18], [19]. Orbifold constructions can be applied in the most general form of toroidal compactifications, i.e. the so called Narain compactifications [20] in which there are different numbers of left and right moving chiral bosons, and in which the constant toroidal background fields include not just the metric and antisymmetric tensor fields $G_{i j}$ and $B_{i j}$ but also a Wilson line $A_{i I}$ where $i, j=1$..n label coordinates on the torus $T^{n}$ and $I$ runs over the rank of the gauge group (either $E_{8} \times E_{8}$ or $S O(32)$ ). It is an interesting question to ask whether these more general Heterotic string compactifications, including target space Wilson lines, have a role to play in understanding quantum Hall systems and their possible generalizations [25].

In this paper we will restrict ourselves to constructing orbifolds based on symmetric (i.e. non-heterotic) toroidal compactifications. Following the approach of [15], our starting point is a chiral edge theory for $n$ bosonic fields $\phi^{i}$, which are taken to transform inhomogenuously under a local $U_{1}$ gauge symmetry in $1+1$ dimensions as $\delta_{\lambda} \phi^{i}=q^{i} \lambda$, with charges $q^{i}$. Their action is given by

$$
\begin{aligned}
S= & \frac{1}{8 \pi} \int d^{2} x G_{i j} \mathcal{D}_{\mu} \phi^{i} \mathcal{D}^{\mu} \phi^{j}+\frac{1}{8 \pi} \int d^{2} x B_{i j} \epsilon^{\mu \nu} \partial_{\mu} \phi^{i} \partial_{\nu} \phi^{j} \\
& -\frac{q^{i}}{4 \pi} \int d^{2} x G_{i j} \epsilon^{\mu \nu} \partial_{\mu} \phi^{j} A_{\nu}-\frac{1}{4 k^{2}} \int d^{2} x F_{\mu \nu} F^{\mu \nu}
\end{aligned}
$$

where in eq(1), $\mathcal{D}_{\mu} \phi^{i}=\partial_{\mu} \phi^{i}-q^{i} A_{\mu}$, with $A_{\mu}$ the $1+1$ dimensional $U_{1}$ gauge field, and the so called "anomaly" term has been included (the third term).

The covariant $U_{1}$ current $J_{\text {cov }}^{\mu}$ obtained from the action $S$ is given by

$$
J_{\text {cov }}^{\mu}=\frac{q^{i}}{4 \pi} G_{i j}\left(\mathcal{D}^{\mu} \phi^{j}+\epsilon^{\mu \nu} \mathcal{D}_{\nu} \phi^{j}\right)
$$

which has the property that it is chiral, i.e. $J_{\text {cov }}^{+} \equiv J_{\text {cov }}^{0}+J_{\text {cov }}^{1}=0$, which follows from imposing the chirality condition $\left(\mathcal{D}_{0}-\mathcal{D}_{1}\right) \phi^{i} \equiv \mathcal{D}_{-} \phi^{i}=0$, with $\mu=0,1$ running over coordinates $t, x$. As was noted in [15], this latter constraint is compatible with the $\phi^{i}$ equations of motion, due to the modified action including the anomaly term. Imposing 
the chirality condition is crucial to identifying the current $J_{c o v}^{\mu}$ with the Hall current, at the edge of a sample in $2+1$ dimensions. Indeed, the Hall conductivity $\sigma_{H}$ is obtained through the anomalous conservation law of $J_{c o v}^{\mu}$

$$
\partial_{\mu} J_{c o v}^{\mu}=-\frac{q^{i} G_{i j} q^{j}}{4 \pi} E=-\sigma_{H} E
$$

where $E=\partial_{0} A_{1}-\partial_{1} A_{0}$ is the $1+1$ dimensional electric field.

In the string theory approach, $\phi^{i}$ label compact coordinates of the string as it moves on the torus $T^{n}$, which has constant metric $G_{i j}$ and antisymmetric tensor background fields $B_{i j}$. The spectrum of such theories is well known to possess duality symmetries 3 [14], which exchange momentum states with winding states, and at the same time transform the background fields $G_{i j}$ and $B_{i j}$. These symmetries therefore map the Hall conductivity $\sigma_{H}$ to new values in general, and this has been exploited in [15] in making connections between various Hall hierarchies, and indeed to relate the integer and fractional QHE. We shall review some details of these results later, when we discuss the situation that applies to orbifolds.

First let us consider applying the orbifold construction to the chiral boson action (11). The presence of gauge fields $A_{\mu}$ again makes this construction not completely equivalent to the string case, and as we shall see later there are important differences. The mode expansion of the fields $\phi^{i}(x, t)$ is

$$
\begin{aligned}
& \phi^{i}(x, t)=\frac{1}{2}\left(\phi_{L}^{i}\left(x_{+}\right)+\phi_{R}^{i}\left(x_{-}\right)\right) \\
& \phi_{L}^{i}\left(x_{+}\right)=\phi_{0}^{i}+x_{+} G^{i j} P_{L j}+\sum_{m>0} \frac{1}{\sqrt{m}}\left[a_{L m}^{i} e^{-i m x_{+}}+h . c\right] \\
& \phi_{R}^{i}\left(x_{-}\right)=\phi_{0}^{i}+x_{-} G^{i j} P_{R j}+\sum_{m>0} \frac{1}{\sqrt{m}}\left[a_{R m}^{i} e^{-i m x_{-}}+h . c\right]
\end{aligned}
$$

with $x_{+}=t+x$ and $x_{-}=t-x$, and $a_{L, R m}^{i}\left(a_{L, R m}^{\dagger i}\right)$ the usual anhilation/creation operators. The left and right moving momentum $P_{L, R j}$ have the expansion

\footnotetext{
${ }^{3}$ It should be noted however, that the gauge fields $A_{\mu}$ are absent in the string approach so that strictly speaking the presence of such fields breaks the duality symmetries. Such a breaking is thus due to the response of the Hall system to a electromagnetic field, rather than by the system itself, as has been argued in [15]
} 


$$
\begin{aligned}
P_{L i} & =\frac{1}{2}\left(2 m_{i}+(G-B)_{i j} N^{j}\right) \\
P_{R i} & =\frac{1}{2}\left(2 m_{i}-(G+B)_{i j} N^{j}\right)
\end{aligned}
$$

Here $m_{i}=\operatorname{spec}\left(p_{i}^{\prime}\right)$ is the allowed spectrum of values of the canonical momentum operator $p_{i}^{\prime}$ for the chiral boson moving on $T^{n}$, and $N^{i}$ are corresponding winding numbers. As in [15], $m_{i}$ is allowed to assume both integral and half odd integral values. Let us assume that $T^{n} \equiv R^{n} / \Lambda$ where the $n$-dimensional lattice $\Lambda$ has a set of basis vectors $e_{a}^{i}, a=1 \ldots n$, so that any lattice vector $v^{i}$ can be decomposed as $v^{i}=\sum_{a} e_{a}^{i} l^{a}$ with integer valued coefficients $l^{a}$. One can also define the dual lattice basis $\tilde{e}_{i}^{a}$ which has the property that

$$
\tilde{e}_{i}^{a} e_{a}^{j}=\delta_{i}^{j} \quad, \quad \tilde{e}_{i}^{a} e_{b}^{i}=\delta_{b}^{a}
$$

The index $i$ is often referred to as labelling the "space" basis, whilst the index $a$ labels the "lattice" basis [18]. Using $e_{a}^{i}$ and its dual, one can express various quantities in either of these bases. For example the metric on the torus $G_{i j}=\tilde{e}_{i}^{a} G_{a b} \tilde{e}_{j}^{b}$ etc. , where $G_{a b}$ is the toroidal metric in the lattice basis. Similar relations hold for other fields.

Working in the lattice basis, the well known formulae for the zero mode (i.e. ignoring the oscillator contributions ) parts of the Hamiltonian $H_{0}$ and generator of $x$ translations, $S_{0}$, are

$$
\begin{aligned}
& H_{0}=\frac{1}{2}\left(P_{L}^{t} G^{-1} P_{L}+P_{R}^{t} G^{-1} P_{R}\right) \\
& S_{0}=\frac{1}{2}\left(P_{L}^{t} G^{-1} P_{L}-P_{R}^{t} G^{-1} P_{R}\right)
\end{aligned}
$$

which can be written in a more compact form as

$$
H_{0}=\frac{1}{2} \mathbf{u}^{t} \boldsymbol{\Xi} \mathbf{u} \quad, \quad S_{0}=\frac{1}{2} \mathbf{u}^{t} \eta \mathbf{u}
$$

where

$$
\mathbf{u}=\left(\begin{array}{c}
\mathbf{n} \\
\mathbf{m}
\end{array}\right), \quad \eta=\mathbf{2}\left(\begin{array}{cc}
\mathbf{0} & \mathbf{I}_{n} \\
\mathbf{I}_{n} & \mathbf{0}
\end{array}\right), \quad \boldsymbol{\Xi}=\frac{\mathbf{1}}{\mathbf{2}}\left(\begin{array}{cc}
(\mathbf{G}-\mathbf{B}) \mathbf{G}^{-1}(\mathbf{G}+\mathbf{B}) & \mathbf{B G}^{-1} \\
-\mathbf{G}^{-1} \mathbf{B} & \mathbf{G}^{-1}
\end{array}\right)
$$


$\mathbf{I}_{n}$ being the identity matrix in $n$ dimensions, and the components of the vectors $\mathbf{n}, \mathbf{m}$ given by $N^{i}$ and $M_{i} \equiv 2 m_{i}$ respectively. Note that all the background $G_{a b}$ and $B_{a b}$ dependence lies totally in $H_{0}$, and that with this choice of basis, the quantum numbers $\mathbf{u}$ are integer valued.

Duality symmetries are all those integer-valued linear transformations of the quantum numbers leaving the spectrum invariant. Denote these linear transformations by $\boldsymbol{\Omega}$ and define their action on the quantum numbers $\mathbf{u}$ as

$$
\mathbf{\Omega}: \mathbf{u} \longrightarrow \mathbf{S}_{\Omega}(\mathbf{u})=\mathbf{\Omega}^{-1} \mathbf{u}
$$

$\Omega$ should satisfy the constraint that $\Omega^{\mathrm{t}} \eta \Omega=\eta$ which immediately implies that $\Omega$ is an element of $O(n, n ; Z)$. The backgrounds $G_{i j}$ and $B_{i j}$ are transformed via the change

$$
\Xi \longrightarrow \Omega^{t} \Xi \Omega
$$

The orbifold is defined as the quotient of the torus by a group of discrete isometries, the point group $P$ which is usually taken to be a cyclic group. In the next section we shall consider a simple two-dimensional orbifold based on the discrete group $Z_{3}$. Let $\theta_{j}^{i}$ be the action of an element of $P$ on the torus coordinate $\phi^{i}$, i.e.

$$
P: \phi^{i} \longrightarrow \theta_{j}^{i} \phi^{j}
$$

it follows that if $P$ is a discrete automorphism of $\Lambda$ then we have

$$
\theta_{j}^{i} e_{a}^{j}=e_{b}^{i} Q_{a b}
$$

where the $n \times n$ matrix $\mathbf{Q}$ is integer valued and forms a representation of $P$. From this it follows there is a natural action of $P$ on the quantum numbers $\mathbf{u}$, namely,

$$
P: \mathbf{u} \longrightarrow \mathbf{R} \mathbf{u} ; \quad \mathbf{R}=\left(\begin{array}{cc}
\mathbf{Q} & \mathbf{0} \\
\mathbf{0} & \mathbf{Q}^{*}
\end{array}\right)
$$

and $\mathbf{Q}^{*}=\left(\mathbf{Q}^{t}\right)^{-1}$.

In considering the applicability of the bosonic theory (11) to the orbifolds, important additional constraints have to be placed on the backgrounds $G_{a b}$ and $B_{a b}$. These follow from demanding invariance of the action $S$ under the point group $P$ which yields

$$
\mathrm{Q}^{t} \mathrm{G} \mathrm{Q}=\mathrm{G} \quad, \quad \mathrm{Q}^{t} \mathrm{~B} \mathrm{Q}=\mathrm{B}
$$


These constraints dictate that the background fields have to have the right "shape" in order to admit a discrete isometry. In the present context, such constraints have important consequences regarding the possible values that the Hall conductivity $\sigma_{H}$ can take, since this depends explicitly on $G_{i j}$, which we shall discuss in more detail shortly. The observant reader will have noticed that the conditions (15) are strictly applicable in the string context, when there is no gauge field $A_{\mu}$ present. However, these conditions will still be applicable, and the action $S$ invariant under the point group $P$ if we demand that under the action of $P$, the gauge fields $A_{\mu}$ remain inert, while the charges $q^{i}$ transform as

$$
P: q^{i} \longrightarrow \theta_{j}^{i} q^{j}
$$

which then implies that the quantity $\mathcal{D}_{\mu} \phi^{i}$ transforms homogeneously under the action of the point group. It can be easily seen that as a consequence of this, together with eqs(13) and (15), the conductivity $\sigma_{H}$ after orbifolding, is just the $P$-invariant Hall conductivity on the torus.

Another important point we must address is the compatibility of the chirality condition we must impose on $\phi^{i}$ namely $\mathcal{D}_{-} \phi^{i}=0$ and the action of the point group $P$. In particular we should hope that the chirality condition still holds under this group action. As was shown in [15], this chirality condition imposes restrictions on the allowed spectrum of zero modes, which can be written in the compact form

$$
\Xi \mathbf{u}=\eta \mathbf{u}
$$

That eq(17) correctly encodes the chirality condition, is clear when one recalls that $H_{0}$ and $S_{0}$ are proportional to the zero mode parts of $\left\langle L_{0}+\bar{L}_{0}\right\rangle$ and $\left\langle L_{0}-\bar{L}_{0}\right\rangle$ respectively, where $L_{0}$ and $\bar{L}_{0}$ are the (diagonal) left and right moving Virasoro generators.

Now it follows from the constraints imposed earlier on $G_{a b}$ and $B_{a b}$ and the definition of the twist matrix $\mathbf{R}$ that

$$
\mathbf{R}^{t} \boldsymbol{\Xi} \mathbf{R}=\boldsymbol{\Xi} \quad, \quad \mathbf{R}^{t} \eta \mathbf{R}=\eta
$$

Using (18) it is easy to verify that the chirality condition is indeed preserved under the action of $P$. This is an important result since otherwise it would be hard to see how the orbifolding process has a role to play in quantum Hall systems, since we know they correspond to chiral edge theories [2],[4],[12],[13],[15]. 
Now we want to consider what happens to the duality symmetries in passing from the toroidal to the orbifold edge theory. Again it is well known from the string approach [18] that in the process of orbifolding, the toroidal duality group $O(n, n ; Z)$ is broken in general to a subgroup $\Gamma$ say, where the elements of $\Gamma$ are those transformations $\Omega$ of $O(n, n ; Z)$ satisfying

$$
[\mathbf{R}, \boldsymbol{\Omega}]=\left[\mathbf{R}^{2}, \boldsymbol{\Omega}\right]=\ldots=\left[\mathbf{R}^{N-1}, \boldsymbol{\Omega}\right]=0
$$

where in eq(19) we have considered the simplified case of a point group $P$ isomorphic to the cyclic group $Z_{N}$, with generator represented by $\mathbf{R}$. Similar conditions, namely that the surviving duality group on the orbifold must commute with all the elements of $P$ holds for more general point groups.

This result will again have important consequences in determining the kinds of filling fractions and how they are related in the orbifold edge model. We shall see how this works in a specific model in the next section.

Finally in order to complete our construction, we need to identify (as in the toroidal case) certain fermionic states in our spectrum, which we want to identify with the electrons at the edge of the Hall sample. This implies also that among such states, we should look for those with charge $-e$. In [15], it was emphasized that these additional constraints give the correct physical interpretation of the various filling fractions obtained by specific choices of the metric $G_{a b}$ and those obtained by acting with duality transformations. In the toroidal case, the system consists of bosons, so fermionic states can only be obtained through solitonic configurations via the chiral vertex operators $V\left(x_{+}\right)=: e^{i M_{L i} \phi_{L}^{i}}:$, where $M_{L i}$ are just the allowed values of the left moving momentum $P_{L i}$. Note that $M_{L i}$ need not be integer in general. In passing to the orbifold, such states will also be present in the untwisted sector $\left(V\left(x_{+}\right)\right.$is invariant under the point group action since the $M_{L i}$ also rotate under the action of $P$ ), although in general there will be additional sectors of the total Fock space of states which will correspond to the fields $\phi^{i}$ satisfying twisted boundary conditions. We will discuss these sectors shortly. In the toroidal approach, taking these various points into account, there are three fundamental constraints that have to be satisfied (here we write them in the lattice basis): 


$$
\begin{aligned}
(\text { i }) \quad(G+B)_{a b} N^{b} & =M_{a} \\
(\text { ii }) \quad G_{a b} N^{b} & =M_{L a} \quad, M_{L a^{\prime}}=-1 \quad \text { for some } a^{\prime} \in(1,2, \ldots n) \\
\text { iii }) & M_{a} N^{a}=2 p+1, \quad p \in Z
\end{aligned}
$$

The first condition $(i)$ in eq(20) is just the chirality condition we met earlier. The second condition implies that the charge of the state created by $V\left(x_{+}\right), q$, which is given by

$$
q=M_{L i} q^{i}=M_{L a} q^{a}
$$

is $-e$ if we choose $q^{a^{\prime}}=e, q^{a}=0, a \neq a^{\prime}$. (Note $a^{\prime}$ takes only one of the values $1, \ldots n$ ). The final condition $(i i i)$, is required in order that $V\left(x_{+}\right)$satisfies anti-commutation relations .

Now we have to consider if these constraints are compatible with the point group action. We have already seen that condition $(i)$ is preserved under $P$. Since $(i i i)$ may be written in the form $\mathbf{u}^{t} \eta \mathbf{u}=2 p+1$ then this is also compatible since $\eta$ is invariant under $P$. Condition $(i i)$, like $(i)$ is not invariant but rather both sides of the equation transform in the same way. However the purpose of this condition was as we noted, to enforce that at least some particles in the spectrum have charge $q=-e$. But in fact we see that the charge $q$ defined in (21) is invariant under $P$ since we saw previously that it was crucial that the charges $q^{a}$ transform under the point group, otherwise the action $S$ will not be invariant. Note that we are not saying that if $(i)-(i i i)$ are satisfied in the toroidal case, they will automatically be satisfied for the orbifolding. It remains to find choices of backgrounds $G_{a b}$ and $B_{a b}$ compatible with the point group $P$ which explicitly satisfy these conditions. But once such a solution is found, the action of the point group will preserve it.

We now return to the issue concerning the existence of twisted sector states when one couples the bosonic theory to a world-sheet $U_{1}$ gauge field as we have described in section 1. Without such couplings, the fields $\phi^{i}(z, \bar{z})$ satisfy boundary conditions $\phi^{i}\left(z e^{2 \pi i}, \bar{z} e^{-2 \pi i}\right)=\left(\theta^{p}\right)_{j}^{i} \phi^{j}(z, \bar{z})+2 \pi v^{i}, \quad p=0,1, . . N-1$ where $N$ is the order of the point group ( $Z_{N}$ in this example) and $v^{i} \in \Lambda$. The untwisted sectors correspond to $p=0$ and satisfy the usual toroidal boundary conditions. The other values of $p$ define the so called twisted sectors, and they are characterized by oscillators whose 
mode numbers are fractional, compared to the integral ones defined in the expansions eq(凹), appropriate to the untwisted sector.

Now when one allows $\phi^{i}$ to transform under a local $U_{1}$ gauge transformation, the gauge invariant action (目) implies that in general, $\phi^{i}(z, \bar{z})$ with non-vanishing charges $q^{i}$ are not allowed to have twisted boundary conditions as described above, because this is incompatible with its transformations under the local $U_{1}$. This can be seen from the fact that the Lagrange density will not be a single valued function as we encircle a point in the complex plane, which is required if it is a local density. This is not the case when the $U_{1}$ is absent, since then $\theta_{j}^{i}$ factors coming from the twisted boundary condition on each $\phi^{i}$ cancel. Thus it is peculiar to the coupling of $\phi^{i}(z, \bar{z})$ with $A_{\mu}$, that the effect of orbifolding the toroidal theory with $Z_{N}$ group is to place restrictions on the various backgrounds $\mathbf{G}, \mathbf{B}$ ( and Wilson lines $A_{i I}$ if they are present ) and not to generate twisted sectors. The only exception to this is the case of $Z_{2}$ orbifolds, since in principle the single $U_{1}$ gauge field $A_{\mu}$ could satisfy either periodic or antiperiodic boundary conditions, and this might allow twisted sectors to occur for non-vanishing charges, depending on how the $Z_{2}$ point group is realized on $\phi^{i}$. Further investigations of these possibilities would be interesting.

Twisted sectors are allowed for fields $\phi^{i}$ whose charges are zero. But such chargeless fields decouple from the current $J_{\text {cov }}^{\mu}$ and would seem not to affect directly quantities of interest like $\sigma_{H}$ etc. However there is a subtle an interesting way in which they can influence what the effective duality symmetry that acts on $\sigma_{H}$ can be. This can happen in higher dimensional orbifolds $(n>2)$, where it is possible that for some point groups, an element $\theta^{p}$ say, has a fixed plane as a opposed to a fixed point, associated with it. If we imagine going to a block-diagonal basis then one has $\theta^{p}=$ $\operatorname{diag}\left(\theta^{(1)}, \theta^{(2)}, \ldots \mathbf{I}_{2}, . . \theta^{(r-1)}, \theta^{(r)}\right)$, in the $n=2 r$-dimensional case, where $\mathbf{I}_{2}$ acts on say the $j$ th plane, and the $2 \times 2$ matrices $\theta^{(m)}$ act on separate planes. Now if the charges of $\phi^{i}$ were all zero except $\phi^{i=j}$ and $\phi^{i=j+1}$, then effectively we have $2 r-2$ fields satisfying twisted boundary conditions, whilst the latter two fields just satisfy the usual toroidal boundary conditions for compactification on a 2-torus. Thus essentially from the point of view of the fields that couple to $A_{\mu}$ it looks like we are back to the $n=2$ example discussed earlier, where we might anticipate a $P S L(2, Z) \times P S L(2, Z)$ duality group. However this is not quite correct in general. This is because the $T^{2}$ being picked out in the above example is a subspace of the total $T^{n}$. How this embedding is realized (e.g. if the $n$-dimensional lattice $\Lambda_{n}$ is decomposable as $\Lambda_{n-2} \oplus \Lambda_{2}$, with $\Lambda_{2}$ the lattice 
associated with $T^{2}$, or if $\Lambda_{n}$ is not decomposable in this way) will determine the actual duality symmetry acting in these directions. If the fixed plane $T^{2}$ is associated with sublattice $\Lambda_{2}$, then the duality symmetries are associated with $P S L(2, Z)$ otherwise it is generically a subgroup. This property is well known from the string literature, in particular there are many examples [23], [24] where the effective duality symmetry associated with the $T^{2}$ consist of so called congruence subgroups, $\Gamma_{0}(p)$ and $\Gamma^{0}(p)$ of $P S L(2, Z)$ where

$$
\begin{aligned}
& \Gamma^{0}(p)=\left(\begin{array}{ll}
a & f \\
c & d
\end{array}\right) \quad a d-f c=1, \quad f=0 \bmod p \\
& \Gamma_{0}(p)=\left(\begin{array}{ll}
a & f \\
c & d
\end{array}\right) \quad a d-f c=1, \quad c=0 \bmod p
\end{aligned}
$$

As an illustration of these ideas, table 1 lists the known 6-dimensional non-decomposable $Z_{N}$ orbifolds and their corresponding lattices. 1 The second column in this table gives the eigenvalues $\left(\xi_{1}, \xi_{2}, \xi_{3}\right)$ of the diagonalized point group generators written in the form $\theta=\left(e^{2 \pi i \xi_{1}}, e^{2 \pi i \xi_{2}}, e^{2 \pi i \xi_{3}}\right)$. In table 2 the corresponding duality groups acting on the various $T$ and $U$ moduli associated with particular fixed planes are listed (see [19] for further details).

A consequence of all this is that in edge theories corresponding to non-decomposable orbifolds, generally there will be a restriction on the allowed $P S L(2, Z)$ elements that one may use to generate new Hall fractions by acting on $\sigma_{H}$ compared to t he toroidal case studied in [15]. One might expect this phenomenon to generalize to the case where some point group elements have fixed subtori $T^{2 p} p>2$ associated with them, in which case the duality group would be a subgroup of $O(p, p ; Z)$. In passing, it is interesting to note that congruence subgroups have been suggested as playing an important role in the understanding of various Hall hierarchies [5], [6] so it would be worthwhile to investigate further orbifold models of the type discussed above.

Before moving onto the next section, it is worthwhile recasting the previous orbifold construction, within the Chern-Simons framework, since this has proved a particularly

\footnotetext{
${ }^{4} 6$-dimensional orbifolds are important in compactifications of 10-dimensional superstring theory to $d=4$, which is why they have particular relevance. However, in the present context we can be more general, and can consider $n$-dimensional non-decomposable lattices
} 
Table 1: Examples of 6-dimensional non-decomposable orbifolds and their corresponding lattices

\begin{tabular}{|c|c|c|}
\hline Orbifold & Point group generator & Lattice \\
\hline$Z_{4}-a$ & $(1,1,-2) / 4$ & $S U(4) \times S U(4)$ \\
$Z_{4}-b$ & $(1,1,-2) / 4$ & $S U(4) \times S O(5) \times S U(2)$ \\
$Z_{6}-I I-a$ & $(2,1,-3) / 6$ & $S U(6) \times S U(2)$ \\
$Z_{6}-I I-b$ & $(2,1,-3) / 6$ & $S U(3) \times S O(8)$ \\
$Z_{6}-I I-c$ & $(2,1,-3) / 6$ & $S U(3) \times S O(7) \times S U(2)$ \\
$Z_{8}-I I-a$ & $(1,3,-4) / 8$ & $S U(2) \times S O(10)$ \\
$Z_{12}-I-a$ & $(1,-5,4) / 12$ & $E_{6}$ \\
\hline
\end{tabular}

Table 2: Duality groups associated with the moduli of certain fixed planes in nondecomposable $Z_{N}$ orbifolds

\begin{tabular}{|c|c|}
\hline Orbifold & Duality group \\
\hline$Z_{4}-a$ & $\Gamma_{T_{3} / 2}=\Gamma^{0}(2), \quad \Gamma_{U_{3}}=P S L(2, Z)$ \\
$Z_{4}-b$ & $\Gamma_{T_{3}}=\Gamma^{0}(2), \quad \Gamma_{U_{3}}=\Gamma_{0}(2)$ \\
$Z_{6}-I I-a$ & $\Gamma_{T_{3}}=\Gamma^{0}(3), \quad \Gamma_{U_{3}}=\Gamma_{0}(3), \quad \Gamma_{T_{1} / 2}=P S L(2, Z)$ \\
$Z_{6}-I I-b$ & $\Gamma_{T_{3}}=\Gamma^{0}(3), \quad \Gamma_{\left(U_{3}+2\right)}=\Gamma^{0}(3), \quad \Gamma_{T_{1}}=P S L(2, Z)$ \\
$Z_{6}-I I-c$ & $\Gamma_{T_{3}}=\Gamma^{0}(3), \quad \Gamma_{U_{3}}=\Gamma_{0}(3), \quad \Gamma_{T_{1}}=P S L(2, Z)$ \\
$Z_{8}-I I-a$ & $\Gamma_{T_{3}}=\Gamma^{0}(2), \quad \Gamma_{U_{3}}=\Gamma_{0}(2)$ \\
$Z_{12}-I-a$ & $\Gamma_{T_{3} / 2}=P S L(2, Z)$ \\
\hline
\end{tabular}


important probe for studying the physics associated with quantum Hall systems in the past. First we remind the reader that the compactified chiral boson edge theory, which is the starting point for our construction, can be understood in terms of a $U_{1}^{n}$ ChernSimons gauge theory, with $n(2+1)$-dimensional gauge field (1-forms ) $\mathbf{V}^{i} i=1 \ldots n$ where the latter are taken to describe pure gauge degrees of freedom $\phi^{i}$. 目 The $2+1-$ dimensional action is

$$
K \cdot S_{C S}^{\left[U_{1}^{n}\right]}[\mathbf{V}]=\sum_{i j} \frac{K_{i j}}{8 \pi} \int_{\mathcal{M}} \mathbf{V}^{i} \wedge d \mathbf{V}^{j}
$$

with $K_{i j}$ a real valued matrix. In [26], [27] it was shown that the above theory induces a conformal field theory on the boundary $\Sigma=\partial \mathcal{M}$ given by

$$
S[\phi]=\frac{1}{8 \pi} \int_{\Sigma} d^{2} x K_{i j} \partial_{\mu} \phi^{i} \partial_{\nu} \phi^{j}
$$

where in eq(24) we have used the notation introduced at the beginning of this section. Now writing $K_{i j}=G_{i j}+B_{i j}$ we see that the boundary action (24) is equivelant to the ungauged version of the original action we started with eq(20). In deriving this we note that although the antisymmetric tensor term in eq(24) is proportional to the $1+1$ dimensional metric $g^{\mu \nu}$, in fact the difference between this term and the one involving $\epsilon^{\mu \nu}$ in (1) is proportional to the constraint $\partial_{-} \phi^{i}$. Thus we see that the symmetric part of $K_{i j}$ can be associated with the metric on a torus $T^{n}$ which explains the connection between the above Chern-Simons theory and toroidally compactified string theory. Of course in the quantum Hall context we are interested in the gauged version of the action (24) as we saw earlier. Coupling the Chern-Simons theory to the $2+1$ dimensional electromagnetic gauge field 1-form $\mathbf{A}$ can be achieved in the following way [28]

$$
S[\mathbf{V}, \mathbf{A}]=K \cdot S_{C S}^{\left[U_{1}^{n}\right]}[\mathbf{V}]+\sum_{i} \int_{\mathcal{M}}\left(\mathbf{V}^{i} \wedge \tilde{J}_{i}+\frac{q_{i}}{2 \pi} \mathbf{A} \wedge d \mathbf{V}^{i}\right)
$$

where $\tilde{J}_{i}$ are the dual of the quasi-particle current densities $J_{i}$, and the second term represents a minimal coupling of $\mathbf{A}$ to the topological current $d \tilde{\mathbf{V}}^{i}$. As was argued in [28], integrating out the $\mathbf{V}^{i}$ fields one finds that the corresponding filling fraction

\footnotetext{
${ }^{5}$ Here we follow the presentation in [28]
} 
for the quasi-particles is equal to the one implied by eq(3) in the gauged chiral boson system.

Now to obtain the orbifold edge theory in this formalism, we "mod out" the above model with a discrete isometry of the torus $T^{n}$ whose metric is the real part of $K_{i j}$. The action of this point group $P$ on the gauge field 1 -forms $\mathbf{V}^{i}$ will be

$$
P: \mathbf{V}^{i} \longrightarrow \theta_{j}^{i} \mathbf{V}^{j}
$$

Since $\phi^{i}$ is identified as the pure gauge degrees of freedom in $\mathbf{V}^{i}$, this transformation of $\mathbf{V}^{i}$ implies the correct point group action of $\phi^{i}$ we used earlier in eq(12).

Demanding that the ungauged action eq(23) be invariant with respect to this symmetry implies that the matrix $K_{i j}$ must be $P$-invariant

$$
\left(\theta^{t}\right)_{i}^{l} K_{l m} \theta_{j}^{m}=K_{i j}
$$

which just reproduces the $P$-invariant conditions on the background fields $\mathbf{G}$ and $\mathbf{B}$ we saw earlier. For this symmetry to extend to the gauged action eq(25), we require that the charges $q^{i}$ and the quasi-particle current densities $J_{i}$ transform homogeneously under the group $P$ as in eq(16).

Now regarding the possibility of allowing for the fields $\mathbf{V}^{i}$ to satisfy twisted boundary conditions on $\Sigma$ i.e.

$$
\mathbf{V}^{i}\left(z e^{2 \pi i}, \bar{z} e^{-2 \pi i}\right)=\left(\theta^{p}\right)_{j}^{i} \mathbf{V}^{j}(z, \bar{z})
$$

(where the $\mathbf{V}^{i}$ are assumed to be restricted to the boundary $\Sigma$ ), the situation is really equivelant to the arguments we gave earlier when working within the chiral boson formulation. Here we see that the Chern-Simons action will not be well defined in general if we allow the above boundary conditions for non-trivial matrix $\theta$. Furthermore it is the terms in eq(25) that minimally couple $\mathbf{A}$ to the gauge field $\mathbf{V}^{i}$ (i.e.those fields which contribute to the topological currents $d \tilde{\mathbf{V}}^{i}$ ) that force this conclusion upon us, just as in our previous discussions this difficulty arose for fields $\phi^{i}$ with non-vanishing charges $q^{i}$. As then, there are some caveats to these conclusions which could allow for possible twisted sectors for $Z_{2}$ valued twists, if the $U_{1}$ gauge field satisfies appropriate boundary conditions.

Having developed a general framework which extends the previous constructions to the case when the chiral edge theory involves scalars $\phi^{i}$ living on an $n$-dimensional orbifold rather than torus $T^{n}$, later we will consider in detail an illustrative example of 
the ideas presented here. We will see that this example does not fulfill all the physical requirements we would like, but it has the virtues of being a simple toy model which nevertheless illustrates the procedures involved. Given the huge and rich variety of orbifolds that exist, many of which have been investigated in the string literature, it is hard to imagine that examples cannot be found which have closer links with real quantum hall systems. Research towards this end is currently underway [25].

In the next section we shall consider how the chiral orbifold edge theories we have been discussing might be connected to generalizations of so called chiral Luttinger liquids, which appear in the fermionic description of quantum Hall systems.

\section{Connections between orbifold edge theories and generalized Luttinger Liquids}

A system of many fermions at low temperatures, in the normal state, in $3 \mathrm{~d}$, is described by the Landau-Fermi theory. According to the theory, there is a one to one correspondence between the quasi-particle excitations of the interacting system and the excitations of the free fermion gas. The parameters of the theory are phenomenological and are extracted from experiments. In 1d, this Fermi-liquid picture breaks down [33], [35-36]. For example, the correlation functions have algebraic behaviour with anomalous exponents [10]. One model of interacting fermions with universal features is the Luttinger model [34]. In fact it has been proved [21] that the low energy effective theory of edge excitations in the fractional quantum hall case are described by a chiral Luttinger liquid and the anomalous exponents mentioned above are known. We shall give a very brief review of some properties of LL's, based on the recent comprehensive study presented in [17], (and references therein).

Linearizing the free electron dispersion relation about the two Fermi-points, and then adding local interactions one finds a LL Hamiltonian of the form $H_{0}+H_{\text {int }}$, where:

$$
\begin{aligned}
H_{0} & =\frac{\pi u_{F}}{L} \sum_{n}\left(\mathcal{J}_{n} \mathcal{J}_{-n}+\overline{\mathcal{J}}_{n} \overline{\mathcal{J}}_{-n}\right) \\
H_{\text {int }} & =\frac{\pi}{L} \sum_{n}\left(2 g_{1} \mathcal{J}_{n} \overline{\mathcal{J}}_{n}+g_{2}\left(\mathcal{J}_{n} \mathcal{J}_{-n}+\overline{\mathcal{J}}_{n} \overline{\mathcal{J}}_{-n}\right)\right)
\end{aligned}
$$

where in the above Hamiltonian, $L$ is the perimeter of the circle which the fermions of the LL are confined to, and $g_{1}, g_{2}$ are coupling constants. $u_{F}$ is the Fermi velocity which 
enters the linearized dispersion relation for the 1-d Fermi gas, $\epsilon_{l i n}(k)=u_{F}\left(\alpha k-k_{F}\right)$, with $\alpha= \pm 1$ for the right and left Fermi points labeled $R, L$. Here $k$ is the wave number and $k_{F}$ the Fermi wave number.

The operators $\mathcal{J}_{n}$ and $\overline{\mathcal{J}}_{n}$ are the moments of two commuting $U_{1}$ currents $\mathcal{J}(\sigma)$, $\overline{\mathcal{J}}(\sigma)$

$$
\begin{aligned}
& \mathcal{J}_{n}=\int_{0}^{L} \mathcal{J}(\sigma) e^{+2 \pi i n \frac{\sigma}{L}} d \sigma \\
& \overline{\mathcal{J}}_{n}=\int_{0}^{L} \overline{\mathcal{J}}(\sigma) e^{-2 \pi i n \frac{\sigma}{L}} d \sigma
\end{aligned}
$$

These currents are in turn, related to the $\mathrm{L}, \mathrm{R}$ components of a Dirac fermion fields $\Psi_{R}(\sigma)$ and $\Psi_{L}(\sigma)$ that describes the infinite Dirac sea that is present in the infrared limit of the LL,

$$
\mathcal{J}(\sigma)=: \Psi_{R}^{\dagger}(\sigma) \Psi_{R}(\sigma): \quad, \quad \overline{\mathcal{J}}(\sigma)=: \Psi_{L}^{\dagger}(\sigma) \Psi_{L}(\sigma):
$$

The operators $\mathcal{J}_{n}, \overline{\mathcal{J}}_{n}$ satisfy the usual $U_{1} \times U_{1}$ affine algebra.

The total Hamiltonian, in the form eq(29), is not diagonal in the current operators. This can be remedied by a Bogoliubov transformation which redefines the currents [10] and in terms of new current operators $J_{n}, \bar{J}_{n}$ the Hamiltonian can be written in the diagonal form

$$
H_{t o t}=\frac{\pi u_{S}}{L} \sum_{n}\left(J_{n} J_{-n}+\bar{J}_{n} \bar{J}_{-n}\right)
$$

with $u_{S}$ the modified Fermi velocity

$$
u_{S}=\sqrt{\left(u_{F}+g_{2}\right)^{2}-g_{1}^{2}}
$$

The current and density fields $j(\sigma)$ and $\rho(\sigma)$ of the 1-d model are identified as [10]

$$
\rho(\sigma)=\alpha^{-1 / 2}(J(\sigma)+\bar{J}(\sigma)) \quad, \quad j(\sigma)=u_{S} \alpha^{-1 / 2}(J(\sigma)-\bar{J}(\sigma))
$$

where 


$$
\begin{aligned}
u_{N} & =u_{F}+g_{1}+g_{2} \\
u_{J} & =u_{F}-g_{1}+g_{2} \\
\alpha & =\sqrt{u_{N} / u_{J}}
\end{aligned}
$$

$u_{N}$ and $u_{J}$ being the charge and current velocities of the LL. Note athough a slight misuse of notation, we use the same symbol $\alpha$ in eq(35) as appears in the linearized dispersion relation we saw earlier in the non-interacting case, since it corresponds to the same quantity generalized to the interacting case. Basically the physical properties of LL are controlled by the two parameters $u_{S}$ and $\alpha$.

Now an important feature of the LL Hamiltonian in its diagonal form eq(32) is that it can be given a CFT interpretation, at least in the infrared limit. The current -current form of $H_{t o t}$ is suggestive of a Lagrangian description in $1+1$ dimensions which has 4-Fermi interactions, since we have seen that each current is bilinear in terms of the R,L components of Dirac fermions. The Lagrangian description is just the 1-flavour massless Thirring model based on the group $U_{1}$, with Lagrangian (in Euclidean form space)

$$
\mathcal{L}=i \Psi_{L}^{*} \partial_{u} \Psi_{L}+i \Psi_{R}^{*} \partial_{v} \Psi_{R}-h \Psi_{L}^{*} \Psi_{L} \Psi_{R}^{*} \Psi_{R}
$$

where $u=\tau-\sigma, v=\tau+\sigma$ and $h$ the 4-Fermi coupling . If Clearly the coupling $h$ must be related to the parameter $\alpha$ that controls the coupling strength in the LL. We shall discuss the precise relationship shortly.

An interesting property of the massless Thirring model is that it has been shown to be equivelant to a single bosonic field $\phi(\tau, \sigma)$ compactified on a circle of radius $R$ [29]. This relationship is somewhat subtle as it turns out that to reproduce the full spectrum of the compactified boson, the Thirring fields $\Psi_{L}, \Psi_{R}$ have to satisfy twisted boundary conditions, in general, in the compact coordinate $\sigma$. (Note that this twisting of the "fermion" boundary conditions is not to be confused with the twistings related to orbifold compactifications which we are discussing in this paper, which have to be considered on top of these.) Since the radius $R$ is effectively the only parameter of the boson model, it must be related to the coupling $h$. In [29] it was proven that complete

\footnotetext{
${ }^{6}$ Here we follow the notation of $[29]$
} 
equivalence of spectrum in the two formulations requires $h=\frac{1}{2}\left(R-\frac{1}{R}\right)$, so that $R=1$ corresponds to the usual free-fermion point.

In [17] it was also shown how the LL has a bosonic formulation, again as one might anticipate, the boson is compactified on an circle whose radius $R$ was shown to be given by $R=\sqrt{\alpha}$, so that $h=\frac{1}{2}\left(\sqrt{\alpha}-\frac{1}{\sqrt{\alpha}}\right)$. This is consistent since in the case of a non-interacting LL, $u_{N}=u_{J}$ and so $R=1$

Having reviewed some basic properties of standard LL's, what remains is to discuss their relation to incompressible Hall fluids. Basically the idea is that if one considers such a fluid defined on an annular geometry, or equivalently on a cylinder, then the edge theories along each of the two boundaries can be described by the two chiral sectors of the Luttinger CFT. That such an identification can reproduce the physical properties of Hall fluids can be understood from studying charge transport between the two boundaries in the presence of an adiabatic magnetic field (see [17]). In doing so one can learn that basic features like the transport of an integer or fractional number of electrons from one edge to the other as one increases the magnetic field by multiples of the flux quantum $\Phi_{0}$, can be predicted from the Luttinger CFT . In this way one finds that the filling fraction (which is related to $\sigma_{H}$ in the usual way) is given in terms of the parameter $\alpha$ (or the radius $R$ ) of the LL by $\nu=\alpha^{-1}=R^{-2}$.

At this point we can make contact with the (gauged) chiral boson formalism for studying quantum Hall systems [15] upon which the present paper is based. The additional features that these models incorporate, compared to the bosons we have described above is chirality and coupling to a $U_{1}$ gauge field. But both these can be included in the fermionic picture of LL's. Chirality of the boson theory means focusing on one of the two chiral sectors of the LL and gives rise to so called chiral Luttinger liquids or CLL's. They are examples of rational LL's, that is the parameter $\alpha$ must be rational which immediately follows since the corresponding radius of the compactified boson must also be rational to be consistent with chirality [15]. The second feature, namely coupling to an external electromagnetic field can also be achieved (see [17] and refs. therein).

It is interesting to note that the predicted relation between the radius $R$ and $\nu$ as seen above is the inverse of the prediction we get from the anomalies argument in the gauged chiral boson which gives $\nu=R^{2}$. However since there is a duality symmetry $R \rightarrow \frac{1}{R}$ in the system, the two predictions are equivelant.

Now we consider how the orbifolding procedure we have studied in this paper, 
manifests itself within the fermionic LL picture described above. Clearly one needs to consider the generalization to $n$ bosons, which naturally leads one to $n$-flavour LL models, which would have Hamiltonian similar to those considered in eq(29) or eq(32) with inclusion of an index $i=1 \ldots n$ on all the operators. There would be a correspondingly larger number of possible coupling constants analogous to $g_{1}$ and $g_{2}$. The resulting model would presumably be related in the infrared to the $n$-flavour massless Thirring model which can be written as

$$
\mathcal{L}=i \Psi_{L}^{* i} \partial_{u} \Psi_{L}^{i}+i \Psi_{R}^{* i} \partial_{v} \Psi_{R}^{i}-h_{i j} \Psi_{L}^{* i} \Psi_{L}^{i} \Psi_{R}^{* j} \Psi_{R}^{j}
$$

with a matrix of coupling constants $h_{i j}$. As shown in [29], by assigning suitable boundary conditions to the fields $\Psi_{L}^{i}, \Psi_{R}^{i}$ the various charge sectors of the model can reproduce the states of a set of bosons $\phi^{i}$ identified as coordinates on a torus $T^{n}$, in the presence of metric $G_{i j}$ and antisymmetric tensor $B_{i j}$, which was our starting point in section 1 . The explicit connection between these background fields and the couplings $h_{i j}$ is

$$
\begin{aligned}
h_{i j} & =\frac{1}{4} \mathcal{B}_{a i} \mathcal{D}_{a j} \\
\mathcal{B}^{a i} & =Z^{a i}-\tilde{Z}^{a i}+Y^{a b} Z^{b i} \\
\mathcal{D}^{a i} & =Z^{a i}-\tilde{Z}^{a i}-Y^{a b} Z^{b i}
\end{aligned}
$$

where the real matrices $Z^{a i}\left(Y^{a b}\right)$ are symmetric (antisymmetric) respectively and $\tilde{Z}^{a i}$ is the inverse of $Z^{a i}$. These are related to the backgrounds $G_{i j}$ and $B_{i j}$ in the bosonic model, however, as discussed in [29], there is no unique dependence of $Y_{a b}$ and $Z^{a i}$ on these backgrounds. Rather there are various (equivelant) choices, each of which determines the boundary conditions on the fermionic operators, and indeed whether the latter are to be regarded as free fields or not. As a specific example, (see [29] for details) one such choice is $Z^{a i}=e^{a i}, Y_{a b}=B_{a b}$ in which case the fermions are interacting $\left(h_{i j} \neq 0\right)$. It is clear from this that all the constraints that we must impose on the couplets $(\mathbf{G}, \mathbf{B})$ in order to capture the correct physics of quantum Hall systems, as given in (i) - (iii) of eq(20) can now be viewed as constraints on the corresponding $n$-flavoured LL and its CFT representation eq(37). In addition we need to impose the geometric constraints eq(15) to encode the orbifold constraints. This will impose the following conditions on the Thirring couplings $h_{i j}$

$$
h_{a b}=Q_{a}^{t^{c}} h_{c d} Q_{b}^{d}
$$


where we have written quantities in the lattice basis. Thus an immediate consequence of considering orbifold edge theories to describe Hall plateau's is that there are further restrictions on the allowed form of the LL Hamiltonians compared to the toroidal case.

In the present formulation, we can address the issue concerning possible new boundary conditions that the various fields satisfy in the orbifold case, (i.e.the twisted sectors). Our previous arguments concerning this apply here, i.e. that because of the coupling to the $U_{1}$ gauge fields it is difficult to see how general $Z_{N}, N>2$ twisted boundary conditions can be allowed for the bosons $\phi^{i}$. Note that the bosonization rules

$$
\begin{aligned}
\partial_{u} \phi^{a} & =\frac{1}{4} B_{a i} \Psi_{L}^{* i} \Psi_{L}^{i}=J_{L}^{a} \\
\partial_{v} \phi^{a} & =\frac{1}{4} D_{a i} \Psi_{R}^{* i} \Psi_{R}^{i}=J_{R}^{a}
\end{aligned}
$$

allow for arbitrary twistings of the $\Psi_{L, R}^{i}$ boundary conditions, since the corresponding phase factors just appear as global $U_{1}^{n}$ transformations which leave the currents in eq(40) unchanged. The same is true even if one couples the Thirring model to a $U_{1}$ gauge field (which is equivelant to the gauging that gives the corresponding bosonic model), since this coupling is through the currents themselves. As we mentioned before, these twistings are independent of those normally associated with orbifolds. Orbifold boundary conditions would imply that the currents $J_{L, R}^{i}$ satisfy (in the $Z_{N}$ case)

$$
J_{L, R}^{i}(\sigma+2 \pi)=\left(\theta^{p}\right)_{j}^{i} J_{L, R}^{j}(\sigma)
$$

for $p=1 . . N$. In the ungauged theory, such boundary conditions are permissible if the couplings $h_{i j}$ satisfy the constraint eq(39) and the resulting theory would be equivelant to the bosonic orbifold spectrums (again ungauged) we have discussed in the last section. I Including the coupling to a $U_{1}$ gauge field we are lead to the same conclusions discussed in the last section, here seen in the fermionic picture.

Because of the equivalence of the $n$-flavour Thirring model and bosons on $T^{n}$, the duality symmetry group $O(n, n ; Z)$ of the latter is also a symmetry of the former, and

\footnotetext{
${ }^{7}$ Details of this equivalence in the $n=1$ case (where the orbifold is the line element $S^{1} / Z_{2}$ ) was shown some time ago [22]
} 
should also be a symmetry of the corresponding generalized LL. The same will be true of the orbifold model for the surviving duality subgroup of $O(n, n ; Z)$ discussed in the last section. The LL picture offers an interesting alternative way of seeing how duality acts on the filling fraction $\nu$ of the corresponding Hall systems, and it would be particularly interesting to investigate the details of this [25]. For example, determining the effect of such dualities on charge transport from one edge to the other within LL would offer a physical picture of how dualities are linking states of different filling fractions, in both the toroidal and orbifold models.

Finally, in ending this section, we make some brief comments about the finite temperature case. It has been shown in the past [30] that using CFT models to describe finite temperature Fermi liquids, in the Euclidean time formalism, leads naturally to considering CFT's defined on a 2-torus rather than the complex plane. In CFT and in string theory one is used to the idea that for consistency one has to put constraints on the model one is considering by demanding that the 1-loop partition function, which is related to the Euclidean partition function of the Fermi liquid, be invariant under the action of the modular group of the torus $\tau \rightarrow \frac{a \tau+b}{c \tau+d}$ where $\tau$ is the complex modulus of the 2-torus. In string theory this is necessary for unitarity and to avoid global world sheet anomalies, since such modular transformations are just large coordinate transformations. These worldsheet anomalies are related to various space-time anomalies. However in models where there is no space-time interpretation, imposing modular invariance may not always be appropriate. In [17] it has been argued that for LL's corrresponding to the single boson case, the appropriate choice of boundary conditions on the world sheet torus, and their relative weight, is not dictated by modular invariance but by matching conditions with the LL partition function (a general class of such partition functions, based on extended characters, has been calculated in [32]). This might also be the case for our orbifold edge theories; the correct weights of untwisted/twisted sector boundary conditions on the torus should reproduce the "twisted" generalized LL partition one gets by orbifolding the Thirring model. That these weighted boundary conditions would differ, in general, from the modular invariant ones, means for example that (unlike in the usual orbifold constructions [16]), we may not be forced to project onto $P$-invariant states. 


\section{Quantum Hall fractions in the $Z_{3}$ orbifold: A toy model}

In this section we will study in detail the two dimensional orbifold $T^{2} / Z_{3}$ where $T^{2}$ is the maximal torus associated with the group $S U(3)$. A basis of lattice vectors is given by the set of simple roots $\mathbf{e}_{1}, \mathbf{e}_{2}$ which transform under the $Z_{3}$ point group generator $\theta_{j}^{i}, \quad i, j=1,2$ as $[31]$

$$
\theta \mathbf{e}_{1}=\mathbf{e}_{2} \quad, \quad \theta \mathbf{e}_{2}=-\mathbf{e}_{1}-\mathbf{e}_{2}
$$

which can easily be checked to give the $\mathbf{Q}$ matrix

$$
\mathbf{Q}=\left(\begin{array}{cc}
0 & 1 \\
-1 & -1
\end{array}\right) \quad, \quad \mathbf{Q}^{3}=\mathbf{I}_{2}
$$

Now the $Z_{3}$ invariant forms of the metric and antisymmetric tensor fields are easily calculated to be

$$
\mathbf{G}=\frac{1}{2}\left(\begin{array}{cc}
2 g_{11} & g_{11} \\
g_{11} & 2 g_{11}
\end{array}\right) \quad, \quad \mathbf{B}=\left(\begin{array}{cc}
0 & b \\
-b & 0
\end{array}\right)
$$

where $g_{11}$ and $b$ are real parameters. The surviving duality symmetries acting on these fields can be determined as follows. Firstly, for the theory defined on $T^{2}$, the duality symmetry group is $O(2,2 ; Z)$ which is isomorphic (up to additional $Z_{2}$ factors) to the group $P S L^{\prime}(2, Z) \times P S L(2, Z)$. Each of these $P S L(2, Z)$ factors acts on the 4-dimensional vectors $\mathbf{U}^{t}=\left(N^{1}, N^{2}, M_{1}, M_{2}\right)$ as follows:

$$
\begin{aligned}
& P S L^{\prime}(2, Z) \quad: \quad \mathbf{\Omega}^{-1}=\left(\begin{array}{cc}
d^{\prime} \mathbf{I}_{2} & -c^{\prime} \mathbf{L} \\
b^{\prime} \mathbf{L} & a^{\prime} \mathbf{I}_{2}
\end{array}\right) \\
& P S L(2, Z) \quad: \quad \mathbf{\Omega}^{-1}=\left(\begin{array}{cc}
\mathbf{M} & \mathbf{0} \\
\mathbf{0} & \mathbf{M}^{*}
\end{array}\right) \\
& \mathbf{M}=\left(\begin{array}{cc}
a & -f \\
-c & d
\end{array}\right) \quad, \quad \mathbf{L}=\left(\begin{array}{cc}
0 & 1 \\
-1 & 0
\end{array}\right)
\end{aligned}
$$

where the integers $a, f, c, d$ and $a^{\prime}, b^{\prime}, c^{\prime}, d^{\prime}$ satisfy $a f-b c=a^{\prime} d^{\prime}-b^{\prime} c^{\prime}=1$. Now since the matrix $\mathbf{R}$ in this example generates a subgroup of the $P S L(2, Z)$ defined in (45), 
the unbroken duality group $\Gamma$ in the $Z_{3}$ orbifold, as determined by the conditions (19) is just $P S L^{\prime}(2, Z)$. \&

Defining the complex modulus $T=2\left(b+i \frac{\sqrt{3}}{2} g_{11}\right)$, this surviving $P S L(2, Z)$ acts as

$$
T \rightarrow \frac{a^{\prime} T+b^{\prime}}{c^{\prime} T+d^{\prime}}
$$

and is the familiar T-duality present in compactified string theory.

Now let us consider solving the constraints $(i)-(i i i)$ given in eq(20). We will begin with the choice $M_{L 1}=-1$ and consider alternatives later. One can find the following expressions for $N^{2}, M_{1}, M_{2}$ and $M_{L 2}$ as functions of the parameters $g_{11}, b$ and $N^{1}$

$$
\begin{aligned}
N^{2} & =-2 g_{11}^{-1}-2 N^{1} \\
M_{1} & =-1-2 N^{1} b-2 g_{11}^{-1} b \\
M_{2} & =-\frac{3}{2} g_{11} N^{1}-2-N^{1} b \\
M_{L 2} & =-2-\frac{3}{2} g_{11} N^{1}
\end{aligned}
$$

From which we learn that $g_{11}=2 / p, p \in Z$ and $N^{1}=\alpha^{\prime} p, \alpha^{\prime} \in Z$, and $b=k / p, k \in Z$. Substituting these into the constraint (iii), one finds that

$$
N^{1} M_{1}+N^{2} M_{2}=2 \alpha^{\prime} p+2 p\left(2 \alpha^{\prime}+1\right)+6 \alpha^{\prime 2} p
$$

so that in fact, with the choice $M_{L 1}=-1$ the solution of $(i)$ and $(i i)$ describes bosonic rather than fermionic states, since the spin factor in (48) is even and not odd as we require. Given that this is not quite what we want, are there other solutions? We can instead impose that $M_{L 2}=-1$ (there is no exchange symmetry in eq(20) between $M_{L 1}$ and $M_{L 2}$ so in principle, this choice leads to different solutions.) Getting a charge

${ }^{8}$ Strictly speaking the surviving duality group defined by $(\sqrt{19})$ is $P S L^{\prime}(2, Z) \times Z_{3}$. However, the additional $Z_{3}$ symmetry is not relevant for the purposes of this paper, and does not act on the $T$ modulus as defined above in (46). Indeed this $Z_{3}$ symmetry is a subgroup of the $P S L(2, Z)$ that acts on the complex $U$ - modulus which appears in string compactifications on $T^{2}$, given in terms of the metric by $U=\frac{1}{g_{11}}\left(g_{12}+i \sqrt{\operatorname{det} G}\right)$. When one passes to the orbifold and uses the $Z_{3}$ invariant form of the metric (44), it is apparent that this $U$ modulus is just a fixed complex constant which plays no role in what follows. 
- e state would follow from choosing $q^{i=1}=0, q^{i=2}=e$, whereas previously we would have $q^{i=1}=e, q^{i=2}=0$. In this case one finds

$$
\begin{aligned}
N^{2} & =-g_{11}^{-1}-\frac{1}{2} N^{1} \\
M_{1} & =\frac{3}{4} g_{11} N^{1}-\frac{1}{2}-b g_{11}^{-1}-\frac{b N^{1}}{2} \\
M_{2} & =-1-N^{1} b \\
M_{L 1} & =-\frac{1}{2}+\frac{3}{4} g_{11} N^{1}
\end{aligned}
$$

from which we learn that, writing $N^{1}=s \in Z$, that $g_{11}=2 / t, \quad t \in Z$ with the condition that $s+t \in 2 \mathcal{Z}$. At the same time $t$ must be a divisor of $s$ so we write $s=\rho t$ for some $\rho \in Z$ and then we find that the allowed values of $b$ are $b=\frac{k^{\prime}}{t}$. Finally calculating the spin term $N^{t} M$ we find after substituting the values $N^{1}=\rho t \quad, \quad N^{2}=-\frac{t}{2}(\rho+1), \quad M_{1}=\frac{1}{2}\left(\rho\left(3-k^{\prime}\right)-1-k^{\prime}\right), \quad M_{2}=-1-k^{\prime} \rho$,

$$
N^{t} M=\frac{t}{2}\left(3 \rho^{2}+1\right)
$$

It would appear that demanding $N^{t} M$ be an odd integer has two solutions

$$
\begin{aligned}
3 \rho^{2}+1 & =2 u \quad t, u \in 2 Z+1 \\
t & =2 m \quad m \in 2 Z+1 \quad \rho \in 2 Z
\end{aligned}
$$

However it is impossible to find integers $\rho$ and odd integers $u$ satisfying eq(51)(i) so we will assume the second solution (ii).

We note that using this solution, we have the further condition that $k^{\prime} \in 2 Z+1$. Thus by imposing the condition $M_{L 2}=-1$ we have found the necessary conditions on the various parameters to provide a solution to $(i)-(i i i)$ given in eqs $(20)$. Note that with this choice of $\rho$, one has $M_{L 2} \in Z$.

It is easy to see that with the above choice of charges $q^{i}$ and metric component $g_{11}$, the Hall conductivity $\sigma_{H}$ and the corresponding filling fraction, $\nu$ are given by

$$
\sigma_{H}=\frac{e^{2}}{2 \pi}\left(\frac{2}{t}\right) \quad, \quad \nu=\frac{2}{t}=\frac{1}{m}
$$

As discussed in [15] there is a condition that the inverse metric $\mathbf{G}^{-1} \in Z$ in order that, for example, the edge state wavefunctions have correct analyticity properties. Since we have, in our example, 


$$
\mathbf{G}^{-1}=\frac{1}{3}\left(\begin{array}{cc}
2 t & -t \\
-t & 2 t
\end{array}\right)
$$

we learn that $t$ must be integer multiples of 3 . The fact that $t / 3$ must be integer can be traced to the fact that we are considering a $Z_{3}$ orbifold in the present model, but in general we can expect that for $Z_{N}$ orbifolds, or those with point groups containing $N^{t h}$-order elements, a similar constraint will hold involving $t / N$. This is a particularly interesting and clear difference between the torus and orbifold edge state theories, since in the former there are no such constraints on the integer appearing in the denominator of the filling fractions $\nu$, other than it being odd.

Having found at least one set of values of the fields $b, g_{11}$ and corresponding filling fractions that define chiral edge theories which contain fermionic particles with charge $-e$, our strategy, is to see if we can generate new filling fractions by making use of the surviving duality group $\Omega$ present after the orbifolding procedure. In particular the most stringent conditions on whether or not this can be achieved, will comes from demanding that the transformed "couplet" $\left(\mathbf{B}^{\prime}, \mathbf{G}^{\prime}\right)$ satisfy conditions $(i)-($ iii $)$ of eq(20).

Since in our simple example, $\Gamma=P S L(2, Z)$ we can in fact calculate explicitly the most general form of the new filling fractions obtained in this way. From the transformation properties of the $\Xi$ matrix defined earlier, we have

$$
\begin{array}{ccc}
\left(\mathbf{G}^{-1}\right)^{\prime}= & \mathbf{A}_{2}^{t}\left((\mathbf{G}-\mathbf{B}) \mathbf{G}^{-1}(\mathbf{G}+\mathbf{B}) \mathbf{A}_{2}+\mathbf{B G}^{-1} \mathbf{A}_{4}\right) \\
& +\mathbf{A}_{4}^{t}\left(-\mathbf{G}^{-1} \mathbf{B} \mathbf{A}_{2}+\mathbf{G}^{-1} \mathbf{A}_{4}\right) \\
& \\
\mathbf{B}^{\prime}\left(\mathbf{G}^{-1}\right)^{\prime}= & \mathbf{A}_{1}^{t} & \left((\mathbf{G}-\mathbf{B}) \mathbf{G}^{-1}(\mathbf{G}+\mathbf{B}) \mathbf{A}_{2}+\mathbf{G}^{-1} \mathbf{A}_{4}\right) \\
& +\mathbf{A}_{3}^{t}\left(-\mathbf{G}^{-1} \mathbf{B} \mathbf{A}_{2}+\mathbf{G}^{-1} \mathbf{A}_{4}\right)
\end{array}
$$

where the $2 \times 2$ matrices $\mathbf{A}_{1} \ldots . \mathbf{A}_{4}$ are given in terms of the general element $\omega \in$ $P S L(2, Z)$ as

$$
\omega=\left(\begin{array}{ll}
\mathbf{A}_{1} & \mathbf{A}_{2} \\
\mathbf{A}_{3} & \mathbf{A}_{4}
\end{array}\right) \equiv\left(\begin{array}{cc}
\beta \mathbf{I}_{2} & -\gamma \mathbf{L} \\
\delta \mathbf{L} & \alpha \mathbf{I}_{2}
\end{array}\right)
$$

with $\alpha \beta-\delta \gamma=1$, and the matrix $\mathbf{L}$ was defined earlier. Substituting the values 


$$
\mathbf{G}=\frac{1}{t}\left(\begin{array}{ll}
2 & 1 \\
1 & 2
\end{array}\right) \quad \mathbf{B}=\frac{1}{t}\left(\begin{array}{cc}
0 & k^{\prime} \\
-k^{\prime} & 0
\end{array}\right)
$$

after some algebra one finds

$$
\begin{aligned}
& \mathbf{G}^{\prime}=\frac{1}{2}\left(\begin{array}{cc}
2 g_{11}^{\prime} & g_{11}^{\prime} \\
g_{11}^{\prime} & 2 g_{11}^{\prime}
\end{array}\right) \\
& g_{11}^{\prime}=\frac{4 t}{2 \alpha^{2} t^{2}+2 \gamma^{2}\left(k^{\prime 2}+3\right)-4 \alpha \gamma k^{\prime} t}
\end{aligned}
$$

which yields the most general $\operatorname{PSL}(2, Z)$ transformed filling fraction

$$
\nu^{\prime}=\frac{2}{\alpha^{2} t-2 \alpha \gamma k^{\prime}+\frac{\gamma^{2}}{t}\left(k^{2}+3\right)}
$$

What remains is how to interpret the (quasi)-particles in the edge theory that are responsible for these new fractions. The situation is simplified by the fact that conditions $(i)$ and $($ iii $)$ in eq(20) are satisfied by $\left(\mathbf{B}^{\prime}, \mathbf{G}^{\prime}\right)$ if they are satisfied by the initial couplet $(\mathbf{B}, \mathbf{G})$. (In fact the spin $N^{t} M$ is duality invariant ). This means that the quasiparticles of the transformed fractions will have Fermi statistics if we take the particular solution for $(\mathbf{B}, \mathbf{G})$ given in eq(49). The issue concerning what charges they have is more subtle as the condition $M_{L 1}$ is not invariant but transforms under $\operatorname{PSL}(2, Z)$. In general we can expect that the charges of the transformed states are fractions of $-e$ . This fact has been highlighted in [15], and a priori it may not be unacceptable that certain filling fractions are described by such particles. Having said that, we can try and see if any of the fractions given in eq(58) can correspond to electronic edge states. In the toroidal theory, new fractions were obtained that did have this interpretation. The "trick" in showing this was to note that if one has as a result of a particular duality transformation, $\mathbf{B}^{\prime}=0$, then conditions $(i i)$ and $(i i i)$ are equivelant, and the corresponding couplet will be a solution. In the $T^{2}$ case, a representitive $O(2,2 ; Z)$ transformation that achieves this is (in our notation)

$$
\mathbf{B} \stackrel{\omega^{\prime}}{\longrightarrow} \quad \mathbf{B}^{\prime}=0, \quad \omega^{\prime}=\left(\begin{array}{cc}
\mathbf{I}_{2} & -\mathbf{L} \\
0 & \mathbf{I}_{2}
\end{array}\right)
$$

with the initial couplet 


$$
\mathbf{G}=\frac{1}{2 m p}\left(\begin{array}{cc}
2 p & -1 \\
-1 & m
\end{array}\right), \quad \mathbf{B}=\frac{1}{2 m p}\left(\begin{array}{cc}
0 & 1 \\
-1 & 0
\end{array}\right)
$$

Returning to our example, the most general transformation of the combination $\mathbf{B G}^{-1}$ under $\operatorname{PSL}(2, Z)$ is

$\mathbf{B}^{\prime} \mathbf{G}^{\prime-1}=\frac{t}{3}\left(\begin{array}{cc}\beta \gamma \frac{\left(b^{2} t^{2}+3\right)}{t^{2}}-\alpha \beta b+\alpha \delta-\gamma \delta b & -2 \beta \gamma \frac{\left(b^{2} t^{2}+3\right)}{t^{2}}+2 \alpha \beta b-2 \alpha \delta+2 \gamma \delta b \\ 2 \beta \gamma \frac{\left(b^{2} t^{2}+3\right)}{t^{2}}-2 \alpha \beta b+2 \alpha \delta-2 \gamma \delta b & -\beta \gamma \frac{\left(b^{2} t^{2}+3\right)}{t^{2}}+\alpha \beta b-\alpha \delta+\gamma \delta b\end{array}\right)$

where we remind the reader that $b=k^{\prime} / t$. We can determine $\mathbf{B}^{\prime}$ from eq(61) given the form of $\mathbf{G}^{\prime}$ in eq(57).

If we now consider the solutions to the condition $\mathbf{B}^{\prime}=0$, then we find two constraints

$$
\beta \gamma=0 \quad, \quad \alpha \delta-(\beta \alpha+\gamma \delta) b=0
$$

for which there are two solutions

$$
\begin{array}{ll}
\gamma=0 \quad, & \alpha=\beta= \pm 1, \quad b= \pm \delta \\
\beta=0 \quad, & \gamma=-\delta= \pm 1, \quad b= \pm \alpha
\end{array}
$$

These solutions imply that in $(i) \quad k^{\prime}= \pm \delta t$ and (ii) $\quad k^{\prime}= \pm \alpha t$ respectively, which means that $k^{\prime} \in 2 Z$. However this contradicts the assumption that $k^{\prime} \in 2 Z+1$ which was required to ensure that the edge state described fermionic particles (see below eq(51)). Thus in order to maintain charge $-e$ particles in the edge theory ( setting $\mathbf{B}^{\prime}=0$ being one way of achieving this) we are forced to have bosonic edge states. Conversely if we demand that our original couplet $(\mathbf{B}, \mathbf{G})$ describe charge $-e$ fermions, then we have to accept that in the transformed theory $\mathbf{B}^{\prime} \neq 0$, and the fermionic states in this theory will have fractional charges in general. This situation is clearly very dependent on the particular choice of orbifold model, and given the huge class of orbifolds that have appeared in the string literature (see [31] for a partial classification) it is very hard to imagine that models cannot be found where we can 
have charge $-e$ fermionic edge states in both the original and transformed theories, as in the toroidal case. Work in obtaining examples of this kind is in progress [25].

So let us continue with keeping our general transformed $\left(\mathbf{B}^{\prime}, \mathbf{G}^{\prime}\right)$ and calculate the general expression for the transformed electric charge $q^{\prime}$ of the fermionic edge states that give the filling fractions eq(58) . Using eqs(21) and the transformation properties of $M_{L i}$ the result is

$$
q^{\prime}=e \frac{2 t\left(-\alpha t-\gamma\left(3 \rho-k^{\prime}\right)\right)}{2 \alpha^{2} t^{2}-4 \alpha \gamma k^{\prime} t+2 \gamma^{2}\left(k^{\prime 2}+3\right)}
$$

There are some obvious choice of elements $\omega \in P S L(2, Z)$ (other than the identity) for which the transformed charge $q^{\prime}$ is an integer multiple of $e$. Consider the "axionic shift" elements given by $\alpha=\beta= \pm 1, \gamma=0$ and $\delta \neq 0$. In this case $q^{\prime}=q=-e$ since these shifts do not affect the charge, and furthermore they also leave the filling fraction invariant (its easy to see from eq(58) that $\nu^{\prime}=\nu$ for these shifts.) It is not obvious whether these are the only elements for which $q^{\prime}$ is an integer multiple of $e$, As we anticipated, $q^{\prime}$ given in eq 64 is generically given by fractional multiples of the electronic charge. Finally we look at some particular $\operatorname{PSL}(2, Z)$ elements and the filling fractions they give. Taking for example, $\alpha=0, \quad \gamma=-\delta= \pm 1$ gives the filling fraction and charge

$$
\nu^{\prime}=\frac{2 t}{3+x^{2}} \quad, \quad \frac{q^{\prime}}{e}=\frac{ \pm t(3 \rho-x)}{x^{2}+3}
$$

where in obtaining these equations we have set, without loss of generality, $b=k^{\prime} / t=$ $(\alpha t+x) / t \quad, \quad x \in 2 Z+1$, the latter condition on $x$ ensuring that we have fermionic edge states. Recall that $\rho \in 2 Z$, and $m \in 2 Z+1$. Now taking $x=1$ gives odd integer values of $\nu^{\prime}=m$ corresponding to the integral quantum hall effect, since $t=2 \mathrm{~m}$. Moreover, since we saw earlier that $m$ is required to be an odd integer divisible by 3 , another choice $x=3$ gives arbitrary odd integer filling fractions $\nu^{\prime}=m / 3$. In both these cases the corresponding charges are given by odd integer multiples of $-\frac{1}{2} e$. Unfortunately, quasi-particles carrying fractional charges with even denominators are apparently ruled out by physical data, so the $Z_{3}$ orbifold toy-model under discussion in this section must remain just that. In any case it would be somewhat remarkable if one of the simplest 2-dimensional orbifolds should satisfy all the physical requirements. At least the model illustrates the existence of duality transformations that connect the 
original filling fraction $\nu=\frac{1}{m}$ with states with $\nu^{\prime}=m$ and $\frac{m}{3}$, each case corresponding to a different choice of $b$ field. It also provides positive motivation in searching for other orbifold models wich yield more realistic quasi-particle spectra.

Another notable set of filling fractions are those of the Jain hierarchy [3], which are given by the series

$$
\nu=\frac{1}{2 p+\frac{1}{m}} \quad, \quad \nu=\frac{1}{2 p-\frac{1}{m}}
$$

with $m \in 2 Z+1, p \in Z$. From the general form of the transformed fraction eq(58), taking $k^{\prime}=1, \alpha=\beta=+1, \gamma= \pm 1, \delta=0$ gives $\nu^{\prime}=\left(m \pm 1+\frac{1}{m}\right)^{-1}$ which is a Jain fraction with $p=\frac{m \pm 1}{2}$. Similarly another subset of Jain fractions can be obtained from the values $k^{\prime}=3, \alpha=\beta=+1, \gamma= \pm 1, \delta=0$ gives $\nu^{\prime}=\left(m \pm 3+\frac{3}{m}\right)^{-1}$ which corresponds to $p=\frac{m \pm 3}{2}$. These are Jain fractions by virtue of the fact that the odd integer $m$ is divisible by 3 as we saw earlier.

One may ask what are the most general Jain fractions one can obtain this way. Writing $k^{\prime}=2 w+1, w \in Z$, then we can write

$$
\nu^{\prime}=\frac{1}{2 p+\frac{1}{m^{\prime}}} \quad, \quad p \in Z, \quad m^{\prime} \in 2 Z+1
$$

if

$$
m=m^{\prime} \gamma^{2}\left(w^{2}+w+1\right) \quad, \quad 2 p=\alpha\left(\alpha m^{\prime} \gamma^{2}\left(w^{2}+w+1\right)-\gamma(2 w+1)\right)
$$

Since $m$ and $m^{\prime}$ are both odd integers, then we require $\gamma \in 2 Z+1$. Then one can easily see that the right hand side of the second equation in eq(68) is always an even integer, for arbitrary integer values of $\alpha$ and $w$, and arbitrary odd integers $\gamma$, subject of course to the constraint $\alpha \beta-\gamma \delta=1$. This therefore defines the integer $p$ of the Jain fraction. Whether eqs(67) and (68) constitute a complete parameterization of Jain fractions of the type given in the first part of eq(67) is an interesting question. Certainly the situation in the $Z_{3}$ orbifold case is different than in the $T^{2}$. There by taking different starting values for $\mathbf{G}$ and $\mathbf{B}$, it was shown that the Jain fractions eq(67) can be obtained via certain $O(2,2 ; Z)$ transformations of the original fraction $\nu=\frac{1}{m}$. Since such dualities are a symmetry of the total edge state spectrum, one of the surprising conclusions in [20] is that the fraction $\nu=\frac{1}{m}$ and those of the Jain hierarchy, share the same spectrum.

In our case the connections are more subtle and complex, in particular there will be different values of the odd integers $m$ and $m^{\prime}$ that enter the Haldane and Jain fractions, 
unlike the toroidal case where they are equal. Of course it has to be remembered that this is a toy model, only illustrative of the possible applications of orbifold edge theories as remarked earlier.

\section{Conclusions}

In this paper we have presented a general formalism for investigating the possible role that chiral orbifold CFT and their corresponding duality symmetries, may play in understanding the interconnection between various quantum Hall hierarchies. Considering orbifold edge theories in this way, provides a natural generalization of the previous work in the literature, which has made use of the well known toroidal duality group $O(n, n ; Z)$ present in strings compactified on $T^{n}$. Strings compactified on orbifolds share many features with those compactified on $T^{n}$ as well as introducing new features. One of these features is to place further geometric conditions on the allowed values of the background fields $G_{i j}$ and $B_{i j}$ which affects directly the allowed values of Hall conductivity $\sigma_{H}$ and hence the filling fractions one can obtain by applying duality transformations to the couplet $(\mathbf{G}, \mathbf{B})$. We have derived the general conditions, analogous to the toroidal edge theory, which imply that the states responsible for these filling fractions are fermions carrying charge $-e$.

The connection between chiral orbifold edge theories and Luttinger liquids, and their CFT representation in terms of $n$-flavour massless Thirring models was also investigated. The geometric constraints placed on the background fields $\mathbf{G}, \mathbf{B}$ manifest themselves as constraints on the Thirring model couplings $h_{i j}$. These in turn should place constraints on the various couplings of the $n$-flavour Luttinger model. It would be interesting to investigate the details of this, as one would then learn how the geometric symmetries of orbifolds directly translate into "selection rules" for allowed interactions in the generalized Luttinger model. At the same time, it would be worthwhile investigating charge transport between boundaries in this model, as this would provide an alternative derivation of the kinds of filling fractions one can find in orbifold edge theories.

We illustrated the general formalism presented in this paper, with a simple toy model based on the 2-dimensional $Z_{3}$ orbifold, and have seen that the basic constraints allow a description of Haldane fractions with $\nu=\frac{1}{m}, \quad m$ odd. The most general duality transformed filling fraction $\nu^{\prime}$ was derived and although this toy model has 
the feature that the corresponding transformed edge states carry fractional electronic charges in general, we have seen that integer filling fractions as well as certain subset of Jain's hierarchy can be obtained this way.

As we have mentioned earlier, the fact that these latter states have particular fractional charges that are apparently ruled out by physical data is a consequence of the specific $Z_{3}$ orbifold in this example, and is not a feature that is expected to hold for all orbifolds. Indeed since the known class of orbifolds is vast, and their properties quite diverse, we anticipate that a systematic search should yield models with more physical quasi-particle spectra.

\section{Acknowledgments}

S. Skoulakis would like to thank the Onassis foundation and PPARC for funding, and S. Thomas the Royal Society for financial support.

\section{References}

[1] R.E. Prange and S.M. Girvin, eds., The Quantum Hall Effect Springer-Verlag, New York (1990)(second ed.) and references therein; DasSarma Sanker "Perspectives in Quantum Hall Effects: Novel Quantum Liquids in Low-Dimensional Semiconductor Structures", Wiley, New York, (1996) (and references therein); M. Jonssen, O. Viehweg, U. Fastenrath and J. Hajdu, "Introduction to the Theory of the Integer Quantum Hall Effect" New York : VCH (1994) (and references therein).

[2] X.G. Wen, Topological orders and edge excitations in FQH states, cond-mat/9506066

[3] J.K. Jain, Adv. phys. 41 (1992) 105

[4] J. Frohlich and T. Kerler Nucl. Phys. B354 (1991); J. Frohlich and U.M. Studer, Rev. Mod. Phys. 65, 733 (1993); J. Frohlich and E. Thiran (Zurich THH 93-22) 
; J. Frohlich, U.M. Studer, E. Thiran in Les Houches Summer Scholl Session 62 : Fluctuating Geometries in Statistical Mechanics and Field Theory Les Houches France 2 Aug. -9 Sept 1994, cond-mat/9508062

[5] C.A. Lutken and G.G. Ross, Phys. Rev. B45 (1992) 11837 ; Phys. Rev. B48 (1993) 2500

[6] B. P. Dolan, Duality and the Modular group in Quantum Hall Effect condmat/9805171

[7] E. Witten, Comm. Math. Phys. 121 (1989) 351; S. Elitzur, G, Moore, A. Schwimmer and N. Seiberg, Nucl. Phys. B326 (1989) 108 and references therein.

[8] G. Moore and N. Seiberg Phys. Lett B220 (1990) 422

[9] A.P. Balachandran, G. Bimonte, K.S. Gupta and A. Stern, Int. J. Mod. Phys A7 4655 and 5855 (1992)

[10] F.D.M. Haldane, J.Phys. C:Solid State Phys. 14 (1981) 2585

[11] C.G. Callan, Jr. And J.A. Harvey, Nucl. Phys. B250 (1985) 427.

[12] S.G. Naculich, Nucl. Phys B296 (1988) 837.

[13] F. Wilczek, "Fractional Statistics and Anyon Superconductivity" (World Scientific press 1990) ; M. Stone, Ann. Phys. 207 (1991) 38

[14] A. Shapere and F.Wilczek, Nucl. Phys. B320 (1989) 669; A. Giveon, E. Rabinovici and G. Veneziano, Nucl. Phys. B322 (1989) 167; A. Giveon and M. Rocek, Nucl. Phys. B380 (1992) 128; J. Maharana and J.H. Schwarz, Nucl. Phys. B390 (1993) 3 A.Giveon, M. Porrati and E. Rabinovici, Phys. Rep. 244 (1994) 77

[15] A.P. Balachandran, L. Chandar, B. Sathiapalan, Nucl. Phys. B443 (1995) 465

[16] L. Dixon, J.A. Harvey, C. Vafa and E. Witten, Nucl. Phys. B261 (1985) 678 ; Nucl. Phys. B274 (1986) 285

[17] P. Degiovanni, C. Chaubet, R. Melin, Conformal field theory approach to gapless $1 D$ fermion systems and application to the edge excitations of $\nu=\frac{1}{2 p+1}$ quantum Hall sequences. cond-mat/9711173 
[18] M. Spalinski Phys. Lett B 275 (1992) 47 ; J. Erler, D. Jungnicker and H.P. Nilles, Phys. Lett B 276 (1992) 303

[19] A. Love, W.A. Sabra and S. Thomas, Nucl. Phys B427 (1994) 181

[20] K.S. Narain Phys. Lett. B 169 (1987) 41; K.S. Narain, M.H. Sarmadi and E. Witten, Nucl. Phys. B279 (1987) 369

[21] X.G. Wen Phys. Rev. B411 (1990) 12838.

[22] S.Thomas, Int. J. Mod. Phys. A4 (1989) 2561

[23] P. Mayr and S. Steiberger, Nucl. Phys. B407 (1993) 725

[24] D. Bailin, A. Love, W. Sabra and S. Thomas, Mod. Phys. Lett. A9 (1994) 67 ; Phys. Lett. B320 (1994) 21

[25] S. Skoulakis and S. Thomas, work in progress.

[26] I.I. Kogan, Mod. Phys. Lett. A6 (1991) 501;

[27] L. Copper, I.I. Kogan and K.M. Lee Phys. Lett. B 394 (1997) 67

[28] L. Copper, I.I. Kogan and R.J. Szabo, Mirror Maps in Chern-Simons gauge theory OUTP-97-57P, hep-th/9710179

[29] J. Bagger, N. Seiberg, S. Yankielowicz, Nucl. Phys. B289 (1987) 53

[30] J. Cardy, Nucl. Phys. B270 (1986), 186.

[31] Y. Katsuki, Y. Kawamura, T. Kobayashi, N. Ohtsubo, K. Tanioka, Nucl. Phys. B341 (1990) 611

[32] A. Cappelli and G.R. Zemba, Nucl. Phys. $\quad$ B490 (1997) 595.

[33] I.E. Dzyaloshinskii and A.I. Larkin Sov. Phys. JETP 38 (1974) 202

[34] J.M. Luttinger, J. Math. Phys. 4 (1963) 1154.

[35] A.H. MacDonald in Mesoscopic Quantum Physics, Les houches1994 session LXI.

[36] H.J. Schulz in Mesoscopic Quantum Physics, Les houches1994 session LXI. 